EPJ Web of Conferences 101, 06038 (2015)

DOI: $10.1051 /$ epjconf/ 201510106038

(C) Owned by the authors, published by EDP Sciences, 2015

\title{
A search for circumbinary planets in CoRoT eclipsing binary light curves
}

\author{
Peter Klagyivik ${ }^{1,2, a}$ and Hans J. Deeg ${ }^{1,2}$ \\ 1 Instituto de Astrofísica de Canarias, E-38200 La Laguna, Tenerife, Spain \\ 2 Universidad de La Laguna, Dept. Astrofísica, E-38206 La Laguna, Tenerife, Spain
}

\begin{abstract}
Several transiting circumbinary planets have been found in data of the $\mathrm{Ke}$ pler mission [1-5]. Both CoRoT and Kepler have surveyed similar numbers of stars, and the photometric precision of CoRoT is sufficient that it could detect most of the known circumbinary planets; the main draw-back by CoRoT is the much shorter coverage. Still, there is a high chance that some circumbinary planets may be found in its sample of eclipsing binaries (hereafter EBs). Here we report on an ongoing search for circumbinary planets in the full CoRoT data set.
\end{abstract}

\section{Introduction}

For the CoRoT data, a search for circumbinary planets had previously been done only on a subsample of its first runs [6]. This, and the development of a faster and more sensitive search algorithm motivates us to search the whole CoRoT data-set for the presence of such planets. The task is not easy because of instrumental effects, especially jumps in the CoRoT light curves, which frequently mimic transitlike events. Our algorithm considers the quasi-periodic nature of circumbinary transits as well as their varying transit lengths and transit depths.

\section{Method}

During the search there are several steps which have to be done. The critical points are the correction for instrumental effects (e.g. jumps) and the full removal of the EB light curve. Both jumps and eclipse residuals can easily mimic transits. Main steps of the preparation are as follows:

1. EB light curve removal;

2. Correction for instrumental jumps on the residual light curve;

3. EB period refinement;

4. Correction for jumps again;

5. EB light curve removal refinement.

Once we have a clean light curve we can search for quasi-periodic transits-like events. The sample light curve is populated with box-shaped transits and we fit the whole light curve simultaneously. Since the planet orbits two stars, the times of the transits are not strictly periodic but depend on the orbital phase of the two stellar components. Moreover, the transit duration is a function of the binary orbital phase, too. Therefore we fit the transit length, transit depth and phase shift for each transit separately. Further adjustable parameters are the orbital period of the planet and the epoch. During the planet search we minimize the difference between the observed and the model light curves.

a e-mail: pklagyivik@iac.es 

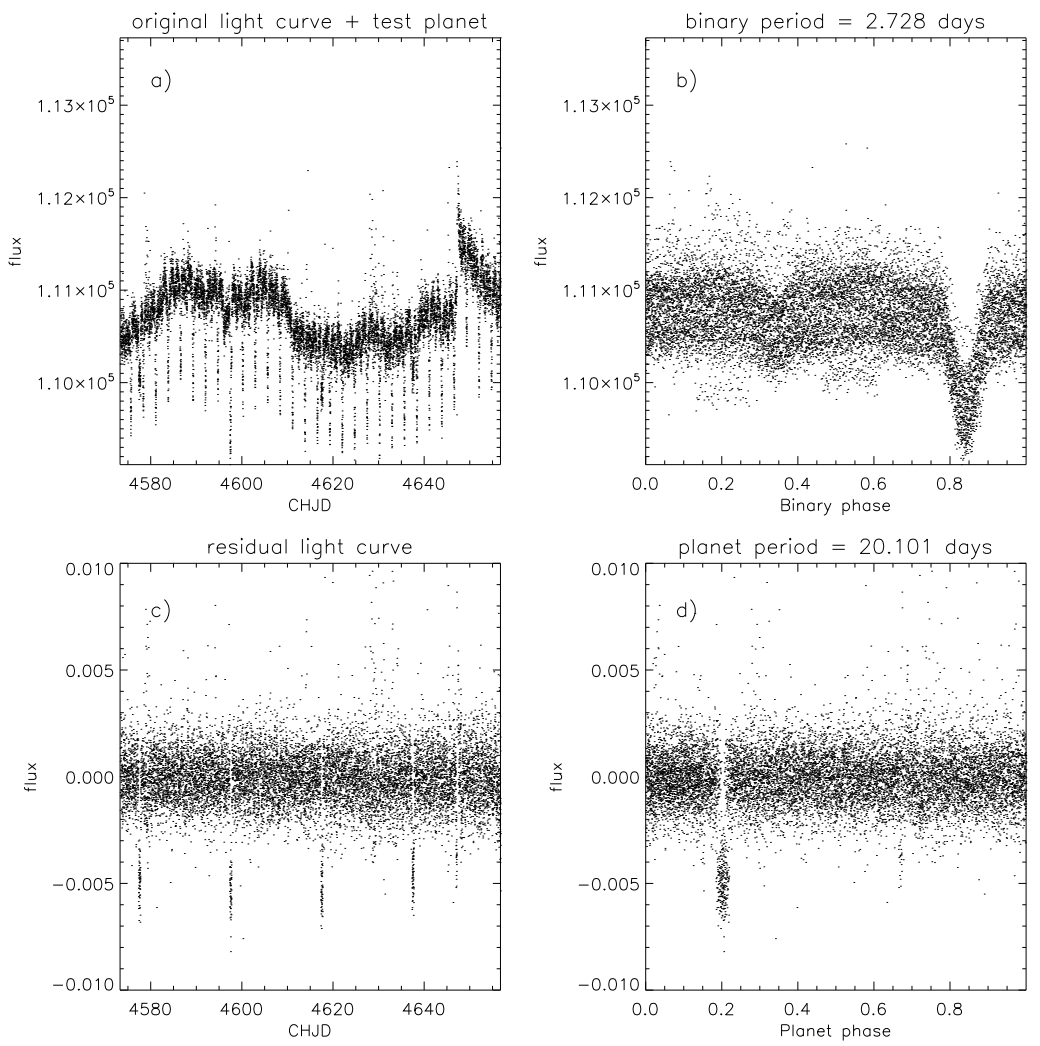

Fig. 1. a) Original light curve with a test planet of $P=20.0$ days. b) Folded binary light curve. c) Residual light curve after the EB removal. d) Folded residual light curve with the planetary period of highest significance.

\section{Results}

First, the code was run on simulated planets. One of these tests is shown in Fig.1, where the planet was successfully detected. The code was also able to retrieve successfully several known Kepler circumbinary planets.

In the case of CoRoT, we searched on all EBs defined in the CoRoT N3 meta-data [7]. Since the N3 classification does not contain the complete set of EBs in CoRoT data, we have extended the sample by EBs identified during the CoRoT planet search. Several candidates are identified. As it was expected all of them are mono-transit events (i.e. there is only a single transit-like event).

Further analysis of the candidates is ongoing. However, improving the handling of instrumental effects might significantly increase the number of candidates. We are also in the process of defining methods to derive useful upper size limits for the non-detection of circumbinary planets.

\section{References}

1. Doyle, L. R. et al., Science 333, (2011) 1602

2. Welsh, W. F. et al., Nature 481, (2012) 475

3. Orosz, J. A. et al., Science 337, (2012) 1511

4. Orosz, J. A. et al., ApJ 758, (2012) 87

5. Kostov, V. B. et al., ApJ 787, (2014) 93

6. Almenara, J. M., PhD thesis (2010)

7. Debosscher, J. et al., A\&A 506, (2010) 519 